\title{
THE PULSATION PROPERTIES OF PROCYON A
}

\author{
Brian Chaboyer \\ Department of Physics and Astronomy, Dartmouth College, 6127 Wilder Laboratory, \\ Hanover, NH 03755-3528; brian.chaboyer@dartmouth.edu \\ P. Demarque \\ Department of Astronomy and Center for Solar and Space Research, Yale University, \\ Box 208101, New Haven, CT 06520-8101; demarque@astro.yale.edu \\ AND \\ D. B. Guenther \\ Department of Astronomy and Physics, Saint Mary's University, \\ Halifax, NS B3H 3C3, Canada; guenther@ap.stmarys.ca \\ Received 1999 August 13; accepted 1999 September 1; published 1999 October 5
}

\begin{abstract}
A grid of stellar evolution models for Procyon A has been calculated. These models include the best physics available to us (including the latest opacities and equation of state) and are based on the revised astrometric mass of Girard et al. Models were calculated with helium diffusion and with the combined effects of helium and heavy-element diffusion. Oscillation frequencies for $l=0,1,2$, and $3 p$-modes and the characteristic period spacing for the $g$-modes were calculated for these models. We find that $g$-modes are sensitive to model parameters that effect the structure of the core, such as convective core overshoot, the heavy-element abundance, and the evolutionary state (main sequence or shell hydrogen burning) of Procyon A. The $p$-modes are relatively insensitive to the details of the physics used to model Procyon A and only depend on the evolutionary state of Procyon A. Hence, observations of $p$-mode frequencies on Procyon A will serve as a robust test of stellar evolution models.

Subject headings: binaries: visual — stars: evolution — stars: individual ( $\alpha$ Canis Minoris, Procyon $)-$ stars: oscillations
\end{abstract}

\section{INTRODUCTION}

Procyon's internal structure makes it a particularly interesting target for seismological studies. Because models of stars at Procyon's position in the H-R diagram have convective envelopes and because we know for the Sun that the solar convection zone is responsible for driving its $p$-mode oscillations, we do expect to see $p$-mode oscillations on Procyon. Furthermore, because Procyon's convective envelope is very thin, any $g$-modes that exist (possibly driven by Procyon's convective core) may be observable at the surface, not being completely damped out as they are in the case of the Sun with its more extensive convective envelope.

Seismology would establish the evolutionary status of Procyon A, which could be either in the core hydrogen-burning phase or in the more advanced hydrogen shell-burning phase, after the core has been exhausted and is composed purely of helium. In the latter case, the presence of an exhausted core and a mean molecular weight discontinuity would leave an unmistakable signature on the $p$-mode frequency spacings due to the existence of mode bumping where the $g$-mode spectrum overlaps (bumps into) and perturbs the $p$-mode spectrum. Mode bumping affects the spacing between adjacent $p$-mode frequencies and, hence, can be discerned from nonbumped $p$ modes. The $g$-mode spectrum would, of course, provide even more direct clues as to the exact nature of Procyon's core.

The Procyon binary system consists of an F5 IV-V primary and a white dwarf secondary in an $40.8 \mathrm{yr}$ orbit. The F5 primary (Procyon A) is a bright, nearby star with a well-determined parallax and astrometric mass. As such, it presents a unique target for the study of nonradial stellar oscillations. Because of the large difference in brightness between the two stars in Procyon, the orbital parameters have been difficult to measure. Strand (1951) derived a mass of $1.75 M_{\odot}$ for Procyon A, a mass that was incompatible with astrophysical estimates from stellar interior models (Guenther \& Demarque 1993) and the spectroscopic $\log g$. Only recently, with improved orbital elements and parallax and a precise angular scale derived using the cold chronograph at the NASA Infrared Telescope Facility (Wang et al. 1994), has it been possible to redetermine the mass of Procyon A (Girard et al. 1996). The revised mass, about $1.5 M_{\odot}$, is now in agreement with the astrophysical mass estimate.

Chaboyer, Demarque, \& Guenther (1998), stimulated by the SONG ground-based campaign (Pilachowski et al. 1998) to detect oscillations on Procyon, made a study of the evolutionary status of Procyon A, using up-to-date physical input and the revised Procyon A mass. Because the SONG data analysis is not yet complete, only a brief summary of these Procyon models was published.

However, the situation has changed dramatically in the last few weeks with the successful identification of individual mode frequencies in the giant star $\alpha$ UMa A by Buzasi et al. (1999), using the small telescope on the Wide Field Infrared Explorer (WIRE) space mission. Because Procyon is on the WIRE target list for observations in the near future, theoretical models and their predicted frequencies have become particularly relevant. We present in this Letter a more detailed description of our interior models and a list of individual $p$-mode frequencies for our reference model. We also include a table of averaged large and small $p$-mode spacings and the characteristic $g$-mode period spacings for our models.

\section{MODELING PROCYON A}

Models for Procyon A were calculated using the Yale stellar evolution code in its nonrotating configuration (Guenther et al. 1992). These models included the latest OPAL opacities (Ig- 
lesias \& Rogers 1996) and the OPAL equation of state (Rogers, Swenson, \& Iglesias 1996). The diffusion coefficients are from Thoul, Bahcall, \& Loeb (1994). Most of the models only included helium diffusion. A single model that included both helium and heavy-element diffusion (treated as a single mean heavy element $Z$ ) was evolved to study the effects of heavyelement diffusion on Procyon A. The models included the effects of a wind mass loss in the diffusion equations. A wind velocity of $v_{w}=-\dot{M} /\left(4 \pi \rho r^{2}\right)$ was included in the diffusion equations, and a solar mass-loss rate $\dot{M}=2 \times 10^{-14} M_{\odot} \mathrm{yr}^{-1}$ was assumed. This wind velocity was large enough to effectively suppress the diffusion in the outer layers of the model. One model was calculated without wind loss. The models employed a solar calibrated mixing length, although the mainsequence models of Procyon A have a very thin surface convection zone and so the structure of these models is largely independent of the mixing length. None of our models include the effects of rotation. Since Procyon A is not a rapidly rotating star, the structure effects of rotation will be much smaller than the other factors considered in this Letter. Rotation likely leads to mixing in stars, and this mixing can suppress diffusion in the outer layers of the star (Chaboyer et al. 1995). This effect is similar to the inclusion of a stellar wind (which also suppresses diffusion in the outer layers), and so a rough estimate of the importance of rotational mixing can be made by comparing the standard case to the no-wind case.

The fundamental properties of Procyon A are $M=1.50 \pm$ $0.05 M_{\odot}$ (Girard et al. 1996), $\pi=00^{\prime \prime 2} 2832 \pm 00^{\prime \prime} 0015$ (Girard et al. 1996), $f_{\oplus}=(18.64 \pm 0.87) \times 10^{-6} \mathrm{ergs} \mathrm{cm}^{-2} \mathrm{~s}^{-1}$ (Smalley $\&$ Dworetsky 1995), and an angular diameter of $\phi=5.51 \pm$ 0.05 mas (Mozurkewich et al. 1991), which imply $L=$ $(7.22 \pm 0.35) L_{\odot}$ and $R=(2.09 \pm 0.02) R_{\odot}$. The central values for the mass, radius, and luminosity were adopted for the standard model of Procyon A (see below). The models for Procyon A were evolved from the zero-age main sequence (ZAMS) until they reached the observed radius. In an iterative procedure, the helium abundance was adjusted in the ZAMS model and the model was reevolved until the model matched the observed radius and luminosity of Procyon A. The starting helium abundance was constrained such that $1.5<\Delta Y / \Delta Z<$ 4. In addition, the final surface abundance of $Z / X$ was constrained to be within 0.1 dex of the solar value $Z / X=$ 0.0245 (Grevesse \& Noels 1993) to satisfy the observed constraint that the surface abundances of Procyon A are near solar (Takeda 1994; Takeda et al. 1996; Kato, Watanabe, \& Sadakane 1996; Yushchenko \& Gopka 1996). Models that met all of the above criteria had their pulsation frequencies calculated using Guenther's nonradial nonadiabatic stellar pulsation program (Guenther 1994).

In total, eight different calibrated models of Procyon A were calculated and pulsed (Table 1). The standard model (the first

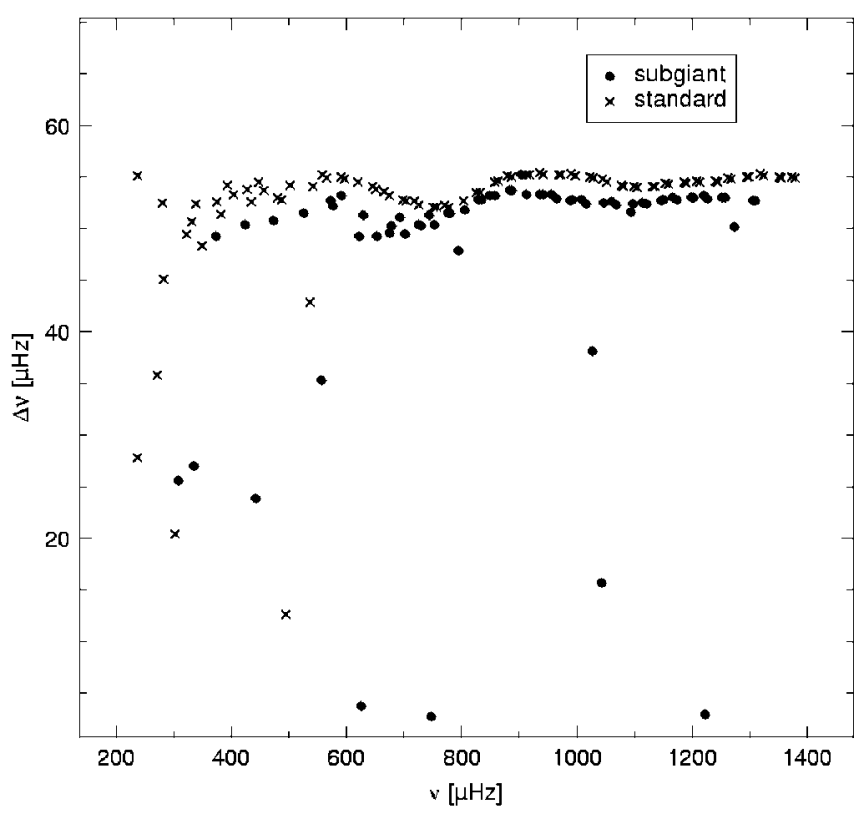

FIG. 1.-Large frequency spacing $\Delta \nu$ plotted as a function of frequency $\nu$ for the standard and subgiant models. Note that irregularities in the large frequency spacings extend to higher frequencies for the subgiant model as compared to the standard model.

line in Table 1) uses the best estimate for the mass, radius, and luminosity, does not include heavy-element diffusion or overshoot at the convective boundaries, and uses our calibrated solar value for the mass fraction of the heavy elements $(Z=0.018)$. The other seven models involved changing a single parameter from the standard model: (1) lower mass (subgiant); (2) evolution to a somewhat lower luminosity (low $L$ ); (3) no wind loss; (4) convective core overshoot of 0.1 pressure scale heights (overshoot); (5) low heavy-element abundance (low $Z$, with $Z=0.015$ ); (6) higher mass (high $M$ ); and (7) heavy-element diffusion ( $Z$ diffusion). All but the subgiant model are in the main-sequence phase of evolution. The lower mass model is in the hydrogen shell-burning phase of evolution.

\section{PULSATION PROPERTIES}

The average first-order frequency spacing $\langle\Delta \nu\rangle$ of the $p$ modes, with $\Delta \nu(n, l) \equiv \nu(n, l)-\nu(n-1, l)$, is likely to be the first quantity determined by stellar seismology. Table 1 includes the frequency spacing averaged over $n=5-25$ for $l=0,1,2$, and 3. The first-order frequency spacing is sensitive to the structure of the outermost layers of the star. Table 1 also includes the average second-order frequency spacing $(\langle\delta \nu\rangle$, averaged over $n=5-25$ ) for $l=0$ and 1 . The second-order

TABLE 1

Model Characteristics

\begin{tabular}{|c|c|c|c|c|c|c|c|c|c|c|c|c|c|}
\hline Model & $\begin{array}{l}\text { Mass } \\
\left(M_{\odot}\right) \\
\end{array}$ & $\Delta Y / \Delta Z$ & $Z / X_{\text {env }}$ & $\begin{array}{l}M_{\text {core }} \\
\left(M_{\odot}\right) \\
\end{array}$ & $\begin{array}{c}M_{\text {scz }} \\
\left(M_{\odot}\right) \\
\end{array}$ & $\log \left(L / L_{\odot}\right)$ & $\begin{array}{c}\left\langle\Delta \nu_{0}\right\rangle \\
(\mu \mathrm{Hz}) \\
\end{array}$ & $\begin{array}{c}\left\langle\Delta \nu_{1}\right\rangle \\
(\mu \mathrm{Hz}) \\
\end{array}$ & $\begin{array}{l}\left\langle\Delta \nu_{2}\right\rangle \\
(\mu \mathrm{Hz}) \\
\end{array}$ & $\begin{array}{l}\left\langle\Delta \nu_{3}\right\rangle \\
(\mu \mathrm{Hz}) \\
\end{array}$ & $\begin{array}{l}\left\langle\delta \nu_{0}\right\rangle \\
(\mu \mathrm{Hz})\end{array}$ & $\begin{array}{l}\left\langle\delta \nu_{1}\right\rangle \\
(\mu \mathrm{Hz})\end{array}$ & $\begin{array}{c}\Pi \\
(\mu \mathrm{Hz}) \\
\end{array}$ \\
\hline Standard & 1.50 & 3.03 & 0.0253 & 0.118 & $1.17 \mathrm{E}-4$ & 0.85868 & 53.95 & 54.15 & 51.38 & 54.40 & 4.75 & 7.01 & 60.58 \\
\hline Subgiant & 1.40 & 2.94 & 0.0239 & 0.000 & $5.62 \mathrm{E}-5$ & 0.85838 & 51.89 & 48.71 & 46.23 & 45.64 & 6.03 & 7.35 & 16.87 \\
\hline Low $L$. & 1.50 & 2.47 & 0.0243 & 0.113 & $2.31 \mathrm{E}-4$ & 0.83747 & 54.19 & 54.34 & 51.62 & 51.80 & 4.69 & 7.06 & 59.35 \\
\hline No wind $\ldots \ldots \ldots$ & 1.50 & 3.12 & 0.0196 & 0.122 & $1.36 \mathrm{E}-5$ & 0.85852 & 52.99 & 53.22 & 50.50 & 50.69 & 5.09 & 7.26 & 62.02 \\
\hline Overshoot ........ & 1.50 & 2.53 & 0.0248 & 0.133 & $1.04 \mathrm{E}-4$ & 0.85848 & 53.80 & 54.06 & 51.28 & 54.25 & 4.64 & 6.92 & 64.77 \\
\hline Low $Z$. & 1.50 & 2.02 & 0.0206 & 0.107 & $1.15 \mathrm{E}-4$ & 0.85860 & 53.87 & 54.05 & 51.41 & 54.52 & 5.23 & 7.10 & 56.35 \\
\hline High $M$ & 1.54 & 1.90 & 0.0247 & 0.121 & $1.07 \mathrm{E}-4$ & 0.85854 & 54.75 & 54.95 & 54.97 & 52.32 & 4.58 & 7.17 & 61.65 \\
\hline$Z$ diffusion ....... & 1.50 & 3.01 & 0.0253 & 0.118 & $1.19 \mathrm{E}-4$ & 0.85847 & 53.94 & 54.15 & 51.38 & 54.40 & 4.73 & 7.01 & 60.70 \\
\hline
\end{tabular}


TABLE 2

Standard Model Frequencies (IN $\mu \mathrm{Hz}$ )

\begin{tabular}{|c|c|c|c|c|c|c|c|c|c|c|c|c|}
\hline$l$ & $n$ & $n_{p}$ & $n_{g}$ & $\nu$ & $\Delta \nu$ & $\delta \nu$ & $l$ & $n$ & $n_{p}$ & $n_{g}$ & $\nu$ & $\Delta \nu$ \\
\hline \multirow[t]{23}{*}{$0 \ldots \ldots$} & 0 & 0 & 0 & 129.02 & $\ldots$ & $\ldots$ & \multirow[t]{24}{*}{$2 \ldots \ldots$} & 0 & 2 & 2 & 208.98 & \\
\hline & 2 & 2 & 0 & 227.83 & $\ldots$ & $\ldots$ & & 1 & 2 & 1 & 236.75 & 27.77 \\
\hline & 3 & 3 & 0 & 280.35 & 52.52 & 7.84 & & 2 & 3 & 1 & 272.51 & 35.76 \\
\hline & 4 & 4 & 0 & 330.93 & 50.59 & 8.96 & & 3 & 4 & 1 & 321.97 & 49.47 \\
\hline & 5 & 5 & 0 & 382.26 & 51.32 & 7.71 & & 4 & 5 & 1 & 374.55 & 52.57 \\
\hline & 6 & 6 & 0 & 434.86 & 52.61 & 6.47 & & 5 & 6 & 1 & 428.39 & 53.84 \\
\hline & 7 & 7 & 0 & 487.66 & 52.80 & 6.26 & & 6 & 7 & 1 & 481.40 & 53.01 \\
\hline & 8 & 8 & 0 & 541.74 & 54.08 & $\ldots$ & & 7 & 7 & 0 & 494.03 & 12.63 \\
\hline & 9 & 9 & 0 & 596.51 & 54.76 & $\ldots$ & & 8 & 8 & 0 & 536.95 & 42.92 \\
\hline & 10 & 10 & 0 & 650.31 & 53.80 & $\ldots$ & & 9 & 9 & 0 & 591.97 & 55.01 \\
\hline & 11 & 11 & 0 & 702.97 & 52.66 & $\ldots$ & & 10 & 10 & 0 & 646.02 & 54.06 \\
\hline & 12 & 12 & 0 & 755.02 & 52.05 & $\ldots$ & & 11 & 11 & 0 & 698.82 & 52.80 \\
\hline & 14 & 13 & 0 & 807.81 & $\ldots$ & 4.21 & & 12 & 12 & 0 & 750.94 & 52.11 \\
\hline & 15 & 14 & 0 & 862.40 & 54.60 & 4.33 & & 13 & 13 & 0 & 803.60 & 52.66 \\
\hline & 16 & 15 & 0 & 917.64 & 55.23 & 4.35 & & 14 & 14 & 0 & 858.07 & 54.47 \\
\hline & 17 & 16 & 0 & 972.79 & 55.16 & 4.35 & & 15 & 15 & 0 & 913.28 & 55.21 \\
\hline & 18 & 17 & 0 & 1027.70 & 54.91 & 4.28 & & 16 & 16 & 0 & 968.45 & 55.16 \\
\hline & 19 & 18 & 0 & 1081.82 & 54.12 & 4.21 & & 17 & 17 & 0 & 1023.42 & 54.97 \\
\hline & 20 & 19 & 0 & 1135.90 & 54.08 & 4.21 & & 18 & 18 & 0 & 1077.61 & 54.19 \\
\hline & 21 & 20 & 0 & 1190.29 & 54.39 & 4.16 & & 19 & 19 & 0 & 1131.70 & 54.08 \\
\hline & 22 & 21 & 0 & 1244.81 & 54.51 & 4.10 & & 20 & 20 & 0 & 1186.13 & 54.44 \\
\hline & 23 & 22 & 0 & 1299.77 & 54.97 & 4.02 & & 21 & 21 & 0 & 1240.71 & 54.58 \\
\hline & 24 & 23 & 0 & 1354.69 & 54.91 & 3.87 & & 22 & 22 & 0 & 1295.76 & 55.05 \\
\hline \multirow{25}{*}{$1 \ldots \ldots$} & -2 & 0 & 2 & 129.55 & $\ldots$ & $\ldots$ & & 23 & 23 & 0 & 1350.82 & 55.06 \\
\hline & 0 & 1 & 1 & 181.62 & $\ldots$ & $\ldots$ & \multirow[t]{24}{*}{$3 \ldots \ldots$} & 2 & 3 & 1 & 286.72 & $\ldots$ \\
\hline & 1 & 2 & 1 & 236.71 & 55.08 & $\ldots$ & & 3 & 4 & 1 & 339.15 & 52.43 \\
\hline & 2 & 3 & 1 & 281.78 & 45.07 & $\ldots$ & & 4 & 5 & 1 & 393.34 & 54.19 \\
\hline & 3 & 4 & 1 & 302.21 & 20.43 & 15.49 & & 5 & 6 & 1 & 447.87 & 54.53 \\
\hline & 4 & 5 & 1 & 350.49 & 48.28 & 11.34 & & 6 & 7 & 1 & 502.07 & 54.20 \\
\hline & 5 & 6 & 1 & 403.82 & 53.32 & 10.47 & & 7 & 8 & 1 & 557.30 & 55.23 \\
\hline & 6 & 7 & 1 & 457.48 & 53.66 & 9.61 & & 9 & 9 & 0 & 612.62 & $\ldots$ \\
\hline & 8 & 9 & 1 & 511.24 & $\ldots$ & $\ldots$ & & 10 & 10 & 0 & 666.24 & 53.62 \\
\hline & 9 & 10 & 1 & 566.13 & 54.89 & $\ldots$ & & 11 & 11 & 0 & 718.88 & 52.65 \\
\hline & 10 & 10 & 0 & 620.65 & 54.52 & 8.03 & & 12 & 12 & 0 & 771.10 & 52.22 \\
\hline & 11 & 11 & 0 & 673.85 & 53.20 & 7.62 & & 13 & 13 & 0 & 824.58 & 53.47 \\
\hline & 12 & 12 & 0 & 726.14 & 52.29 & 7.26 & & 14 & 14 & 0 & 879.65 & 55.08 \\
\hline & 13 & 13 & 0 & 778.19 & 52.04 & 7.09 & & 15 & 15 & 0 & 935.03 & 55.37 \\
\hline & 14 & 14 & 0 & 831.69 & 53.51 & 7.12 & & 16 & 16 & 0 & 990.31 & 55.29 \\
\hline & 15 & 15 & 0 & 886.65 & 54.96 & 7.00 & & 17 & 17 & 0 & 1045.11 & 54.80 \\
\hline & 16 & 16 & 0 & 941.82 & 55.16 & 6.79 & & 18 & 18 & 0 & 1099.24 & 54.13 \\
\hline & 17 & 17 & 0 & 996.92 & 55.10 & 6.61 & & 19 & 19 & 0 & 1153.64 & 54.40 \\
\hline & 18 & 18 & 0 & 1051.45 & 54.53 & 6.34 & & 20 & 20 & 0 & 1208.28 & 54.64 \\
\hline & 19 & 19 & 0 & 1105.40 & 53.95 & 6.17 & & 21 & 21 & 0 & 1263.15 & 54.87 \\
\hline & 20 & 20 & 0 & 1159.70 & 54.30 & 6.06 & & 22 & 22 & 0 & 1318.42 & 55.27 \\
\hline & 21 & 21 & 0 & 1214.21 & 54.51 & 5.93 & & 23 & 23 & 0 & 1373.45 & 55.03 \\
\hline & 22 & 22 & 0 & 1269.01 & 54.80 & 5.86 & & & & & & \\
\hline & 23 & 23 & 0 & 1324.15 & 55.14 & 5.72 & & & & & & \\
\hline & 24 & 24 & 0 & 1378.99 & 54.85 & 5.54 & & & & & & \\
\hline
\end{tabular}

frequency spacings, $\delta \nu(n, l) \equiv \nu(n, l)-\nu(n-1, l+2)$, are sensitive to the structure of the innermost layers of the star. Table 1 includes the characteristic period spacing $\Pi$ of the $g$ modes.

A list of modes from $l=0$ to 3 and $n=0$ to 24 for the standard model is given inTable 2 . In this table, $n_{p}$ is the number of modes in the eigenfunction that are $p$-mode-like (with respect to their phase characteristics) and $n_{g}$ is the number of modes in the eigenfunction that are $g$-mode-like. The gaps in the spacings are due to the $n$-value naming convention (Unno et al. 1989). In regions of variable mean molecular weight, the frequencies of the $g$-modes trapped in the interior may overlap with the frequencies of the $p$-modes trapped in the envelope, "bumping" the $p$-modes and resulting in the mixing of $p$-mode-like modes and $g$-mode-like modes in the $p$-mode eigenfunction. As a consequence of mode bumping, the large spacing varies irregularly in the frequency range where mixed modes (i.e., mode bumping) occurs. In the standard model only the lowest frequency $p$-modes (lowest $n$ ) are mixed, but for the subgiant model all of the nonradial $p$-modes we calculated, up to $n=25$, are mixed. In Figure 1 we show the large spacing for the standard model and for the subgiant model. As just described, we see that the irregularities in the large spacings extend to higher frequencies for the subgiant model than the standard model.

The frequencies and characteristic frequency spacings depend on the radius of the star. To first order, $\delta R_{*} / R_{*} \simeq \delta \nu / \nu$. The radius of Procyon $\mathrm{A}$ is known to within $1 \%$. Thus, the calculated pulsation frequencies are uncertain at the $1 \%$ level due to the error in the radius. In addition, uncertainties in the modeling of the superadiabatic layer (in both the evolution and pulsation calculations) leads to an estimated uncertainty of $\sim \pm 0.5 \%$ in the $p$-mode frequency calculations (see Guenther $\&$ Demarque 1996). The total error associated with the calculated pulsation frequencies shown in Table 1 is approximately $\sim 1.5 \%$. Thus, differences in $\Delta \nu$ greater than $\sim 1 \mu \mathrm{Hz}$ are significant. From Table 1 we see that only the subgiant model has $\Delta \nu$ 's that are significantly different from the others. When the 
large spacing is observed on Procyon, it will immediately tell us whether or not Procyon has reached the subgiant phase of evolution.

The characteristic period spacing (II) of the $g$-modes shows a much larger variation than $\Delta \nu$ (Table 1). Once again, due to errors in the models and radii of Procyon A, only differences in $\Pi$ greater than $\sim 1 \mu \mathrm{Hz}$ are significant. In this context, it is clear that the detection of $g$-modes in Procyon A would allow one to (1) determine its evolutionary status, (2) determine if appreciable overshoot $\left(\geq 0.05 H_{p}\right)$ is occurring at the edge of the convective core in Procyon A, and (3) provide an estimate of the interior metallicity of Procyon A.

\section{SUMMARY}

A grid of stellar evolution models for Procyon A has been calculated and their pulsation properties investigated. The grid of models was chosen to examine the effect of varying the physics used to construct the models (such as the inclusion of overshoot, heavy-element diffusion, or a stellar wind) as well as uncertainties in the observed parameters of Procyon A (mass, luminosity, radius, and heavy-element composition). The properties of these models (including the average frequency spacing for $p$ - and $g$-modes) are listed in Table 1, whileTable 2 presents a list of individual frequencies for our standard model of Procyon A. The predicted $p$-mode frequencies are fairly similar for all models, except for the model that uses a lower $\left(1.4 M_{\odot}\right)$ mass for Procyon A. This model is in the subgiant (shell hydrogen-burning) phase of evolution, while all other models are on the main sequence. If Procyon $\mathrm{A}$ is a main-sequence star, the predicted frequency spacings are fairly regular, while mode bumping leads to irregular frequency spacings in the subgiant star. Since the predicted frequencies are relatively insensitive to changes in the input physics or the assumed mass, radius, luminosity, and heavy-element abundance of Procyon, the detection of $p$-modes on Procyon A will serve as a robust test of stellar evolution theory. Procyon A is on the WIRE target list for observations in the near future. Since WIRE has been successful in detecting individual mode frequencies in the giant star $\alpha$ UMa A (Buzasi et al. 1999), it is hoped that the predictions presented here for the pulsation properties of Procyon A will soon be tested.

This work was supported in part by a Canadian NSERC grant to D. B. G.

\section{REFERENCES}

Buzasi, D., Catanzarite, J., Laher, R., Conrow, T., \& Kreidl, T. 1999, ApJL, submitted

Chaboyer, B., Demarque, P., \& Guenther, D. B. 1998, in ASP Conf. Ser. 135, A Half Century of Stellar Pulsation Interpretation: A Tribute to Arthur N. Cox, ed. P. A. Bradley \& J. A. Guzik (San Francisco: ASP), 67

Chaboyer, B., Demarque, P., Guenther, D. B., \& Pinsonneault, M. H. 1995, ApJ, 446, 435

Girard, T., et al. 1996, BAAS, 28, 919

Grevesse, N., \& Noels, A. 1993, in Origin and Evolution of the Elements, ed. N. Pratzo, E. Vangioni-Flam, \& M. Casse (Cambridge: Cambridge Univ. Press), 15

Guenther, D. B. 1994, ApJ, 442, 400

Guenther, D. B., \& Demarque, P. 1993, ApJ, 405, 298 . 1996, ApJ, 456, 798

Guenther, D. B., Demarque, P., Kim, Y.-C., \& Pinsonneault, M. H. 1992, ApJ, 387,372

Iglesias, C. A., \& Rogers, F. J. 1996, ApJ, 464, 943
Kato, K.-I., Watanabe, Y., \& Sadakane, K. 1996, PASJ, 48, 601

Mozurkewich, D., et al. 1991, AJ, 101, 2207

Pilachowski, C. A., Barden, S., Hill, F., Harvey, J. W., Keller, C. U., \& Giampapa, M. S. 1998, IAU Symp. 185, New Eyes to See inside the Sun and Stars, ed. F.-L. Deubner, J. Christensen-Dalsgaard, \& D. Kurtz (Dordrecht: Kluwer), 319

Rogers, F. J., Swenson, F. J., \& Iglesias, C. A. 1996, ApJ, 456, 902

Smalley, B., \& Dworetsky, M. M. 1995, A\&A, 293, 446

Strand, K. Aa. 1951, ApJ, 113, 1

Takeda, Y. 1994, PASJ, 46, 53

Takeda, Y., Kato, K.-I., Watanabe, Y., \& Sadakane, K. 1996, PASJ, 48, 511

Thoul, A. A., Bahcall, J. N., \& Loeb, A. 1994, ApJ, 421, 828

Unno, W., Osaki, Y., Ando, H., \& Shibahashi, H. 1989, Nonradial Oscillations of Stars (2d ed; Tokyo: Univ. Tokyo Press)

Wang, S.-I., et al. 1994, Proc. SPIE, 2198, 578

Yushchenko, A. V., \& Gopka, V. F. 1996, Pis ma Astronomicheskii Zhurnal, 22,460 